\title{
WIRED DATA TRANSFER USING SMARTPHONE TECHNOLOGY
}

\author{
R.Rajesh ${ }^{1}$, P.Dhanaraja ${ }^{2}$, A.Gomathi ${ }^{3}$ \\ ${ }^{1}$ Student, Computer Science and Engineering, Narasu's Sarathy Institute of Technology, TamilNadu, India \\ ${ }^{2}$ Student, Computer Science and Engineering, Narasu's Sarathy Institute of Technology, TamilNadu, India \\ ${ }^{3}$ Associate Professor, Computer Science and Engineering, Narasu's Sarathy Institute of Technology, TamilNadu, India
}

\begin{abstract}
This paper proposes the speed of the data transfer can be increased by wired connectivity. That means, connecting a Smartphone with another Smartphone using a wire, the data transfer rate increases. This proposal can be considered as the advancement of the Bluetooth, Near Field Communication and Flash transfer regarding the data transfer rate. Moreover, each Smartphone must have inbuilt and very thin wire ranges up to $15 \mathrm{~cm}$ and one Smartphone wire is connected to other and we can transfer the data through the wire. In order to use both the near field Communication and Flash transfer, two Smartphone must be keep very close to each other and Bluetooth also used mostly by keeping mobiles closely. so it is better to use the wired data transfer in Smartphone.
\end{abstract}

Keywords: Wired, Data transfer Speed, Bluetooth, and Near Field Communication.

$* * *$

\section{INTRODUCTION}

We must come with the statement that the better data transmission is present only in security and also in the speed of that data transmits. The Smartphone that we are using nowa-days includes either Bluetooth or NFC. We are using those in our daily life. But there are some disadvantages present in both the Bluetooth and NFC. These are generally used for transferring data. It is hard to transfer the large number of data in minimum number of time. And by using these Bluetooth and NFC less secure though we are using several authentication methods like password authentication etc. Hence it is easy for the hackers to steal the data that we are used, so by using the method of transmitting those data using a wired connection it is easy to transfer the large number of data in a very short range of time and it is highly secure for using the wired connection and no one can't steal the data. Thus the using of wired connection it is better than the using of the Bluetooth and the Near Field Communication.

\subsection{Data Transmission}

The data transfer can be generally defined as the movement of the data in the form of bits between two or more digital devices. [3] While entering the data through the keyboard, each keyed element is encoded by the electronics within the keyboard into an equivalent binary coded pattern, using one of the standard coding schemes that are used for the purpose of the information interchange. To represent all characters of the keyboard, a unique pattern of 7 or 8 bits in size is used. The Use of 7 bits means that 128 different elements can be represented, while 8 bits can represent 256 elements. A similar procedure is followed for the receiver that decodes every received binary pattern into the corresponding character. There are two types of data transmission. It includes Parallel and Serial transmission, in which Serial transmission is classified as Synchronous and Asynchronous transmission.

\subsection{Bluetooth}

Bluetooth $[4][5][6][7][8]$ is wireless protocol that is used to the nearby devices to communicate over the Radio waves. It does this all without needing any additional hardware or software support. Using a special radio frequency in order to data transmission, it creates a short range network. It is very secure and can connect up to eight devices at the same time. The chip that is used for Bluetooth can be plugged into items like computer, digital cameras, mobile Smartphone and faxes. Bluetooth is convenient in certain situations like transferring of files from one mobile Smartphone to another without cables. The distance of data transmission is small in compared to the other modes of wireless communication. Though Bluetooth has many advantages, it also includes some disadvantages like the slow transfer speed makes Bluetooth not an ideal choice for data transfer when faster connection methods are available. The small range is a disadvantage for some who want to use a Bluetooth outside of that 30-feet radius. While Bluetooth is allowed to turn on, it will reduce the battery power of Smartphone.

\subsection{Near Field Communication}

Generally, NFC is a set of standards for the portable devices. The NFC allows those devices to establish peer-to-peer radio communications, passing data from one device to another by touching them very close together.NFC [9][10][11] provides secure communication to all users. It promotes the transfer of data through the safe channels. NFC is a means of sending data over radio waves. In that sense it is similar to $\mathrm{Wi}-\mathrm{Fi}$ or Bluetooth, but unlike those protocols, NFC can be used to 
induce electric currents within passive components as well as just send data. NFC is a set of short-range wireless technologies, typically requiring a distance of $10 \mathrm{~cm}$ or less. NFC operates at $13.56 \mathrm{MHZ}$ and at rates ranging from 106 Kbits/s to $424 \mathrm{Kbits} / \mathrm{s}$. The main disadvantage of this is an expensive technology. NFC sets up more quickly than standard Bluetooth, but has a lower transfer rate than standard Bluetooth. The maximum data transfer rate of NFC (424 Kbits/s) is lower than that of Bluetooth v 2.1(2.1 Mbits/s).

\subsection{Smartphones}

Smartphone's are generally the extensions on normal cell phones. By using the cell phones we can make phone calls and even some have video recording capabilities but it does not contain the GPS capabilities along with huge number of applications. In general, a Smartphone [1][2] will be based on an operating system that allows it to run applications. Apple's iPhone runs ios, Blackberry Smartphone run the Blackberry operating system. Other devices run Google's Android operating system, HP's web operating system and Microsoft windows phone. The Smartphone has more advanced capabilities and also the features in compared to the basic mobile phones. Hence most of the people usually prefer the Smartphone to use. A Smartphone may allow you to create and edit Microsoft office document or at least view the files. It may allow you to download applications, such as personal and business finance managers, handy personal assistance, or well almost anything, or it allow you to edit photos. Now a day's most of the chances of being theft of the mobile phones are increased mostly. so to prevent mobile phones form theft more application are present in the app market. Some personal security application is also present for the women.

In order to stop the risk of sexual assaults and theft and the security applications are helping in people who in dangerous situation. This all technology is applied throughout the physical support in smart phones.

Table -1: Comparison between NFC and Bluetooth

\begin{tabular}{|l|l|l|}
\hline & NFC & Bluetooth \\
\hline Set-up time & $<0.1 \mathrm{~ms}$ & -6sec \\
\hline Range & Up to10cm & Up to 30m \\
\hline Usability & $\begin{array}{l}\text { Human } \\
\text { centric easy, } \\
\text { intuitive, fast. }\end{array}$ & $\begin{array}{l}\text { Data centric } \\
\text { medium. }\end{array}$ \\
\hline Selectivity & $\begin{array}{l}\text { High given } \\
\text { security }\end{array}$ & Who are you? \\
\hline Use cases & $\begin{array}{l}\text { Pay, get } \\
\text { access, share, } \\
\text { initiate } \\
\text { service, easy } \\
\text { set up }\end{array}$ & $\begin{array}{l}\text { Network for data } \\
\text { exchange, head } \\
\text { set }\end{array}$ \\
\hline
\end{tabular}

\begin{tabular}{|l|l|l|}
\hline $\begin{array}{l}\text { Consumer } \\
\text { experience }\end{array}$ & $\begin{array}{l}\text { Touch, wave, } \\
\text { simply } \\
\text { connect }\end{array}$ & $\begin{array}{l}\text { Configuration } \\
\text { needed. }\end{array}$ \\
\hline
\end{tabular}

\section{WDT-WIRED DATA TRANSFER}

"According to our proposal, the data transfer rate in the wire is high in compare to NFC and BLUETOOTH. The Data transfer wire is presented in-built in the Smartphone."

\subsection{Idea}

At the back side of the phone, there is a presence of rolling wire, which is very thin below the camera and it is about $15 \mathrm{~cm}$ long. By dragging the wire, we can connect it with another phone and we transfer the data. By using this wire, the thickness of the mobile does not change and it fits correct to the phone. Consider an example, a music folder which is $120(\mathrm{MB})$ memory and a rocking songs folder that is present inside the music folder which contains 4 songs and it is about $36 \mathrm{mb}$ memory. If you want to send the 4 songs to another mobile by using NFC is not possible to send the files of about up to $4096 \mathrm{~kb}(4 \mathrm{mb})$ only. Then by using Bluetooth is possible but it takes longer time to transfer the files and mainly the phone charge will reduces. But, as per our concept first drag the wire roll present in the phone and fix it with another phone. Drag the folder and send that via the wire to another phone. Then the phone will shows the percentage of receiving files. After that receiving process gets complete, the 4 songs of $(36 \mathrm{mb})$ will present in the receiving files folder of the memory card. Before receiving the files, the receiver phone shows the option of phone and SD memory, the user can select either the phone or SD memory. Suppose, user wants to send an app to another phone, he can select the phone memory. This is possible by the presence of the inbuilt wired connection setting in the phone. Hence by using our idea, the time consumption is less and large memory files can be transmitted easily, the phone charge won't reduce the data transfer rate is also high in compare to NFC and BLUETOOTH. 


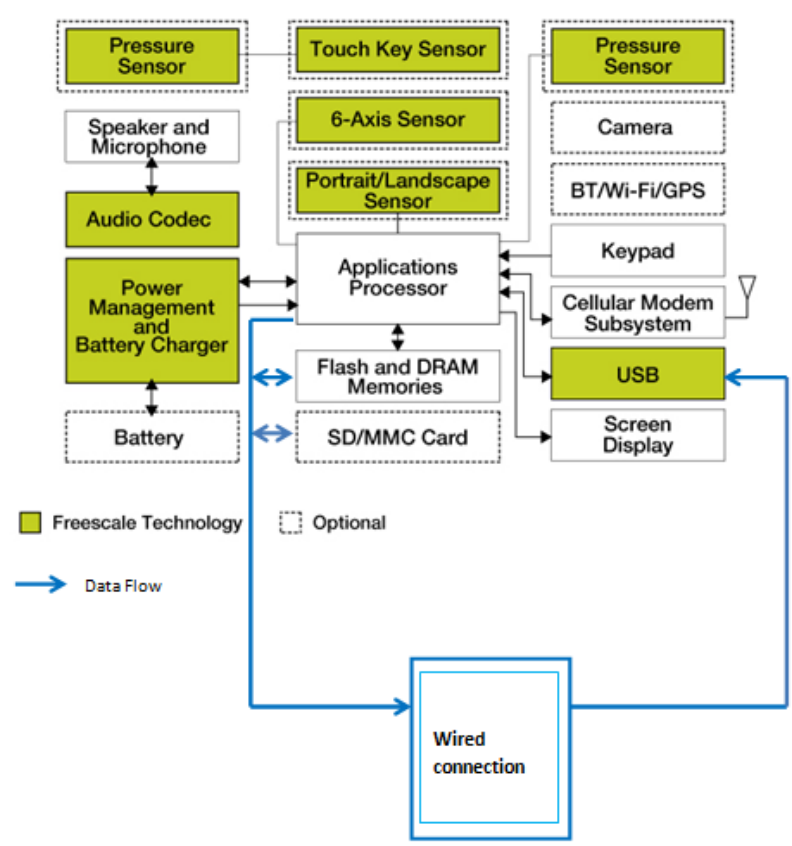

Fig -1: Wired Data Transfer-Smartphone Architecture of Sent a data

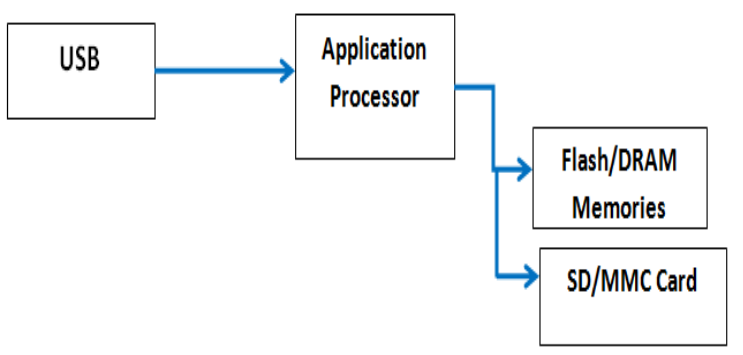

Fig -2: Wired Data Transfer-data receiving Architecture

\section{CONCLUSIONS}

From this we want to conclude that the working with wired connection in the mobiles for the purpose of data transfer is generally good in compare to the Bluetooth and NFC since it has many advantages as mentioned above. While in using flash transfer, it needs flash transfer application software and internet facility in that Smart phones and in NFC also is supported in same company phones only. But in Wired Data transfer smart phone technology no need of internet and WI-FI connection or facilities. According to Wired data transfer Smartphone technology is better idea from comparing to other technologies.

\section{REFERENCES}

[1].http://www.qrcodescanning.com/smartphonehist.html [2].http://cellphones.about.com/od/smartphonebasics/a/what _is_smart.htm

[3].http://ecomputernotes.com/computernetworkingnotes/ ommunication-networks/data-transmission

[4].http://en.wikipedia.org/wiki/Bluetooth

[5].http://www.howtogeek.com/176437/bluetooth-lowenergy-explained-how-types-of-wireless-gadgets-are- nowpossible/

[6].http://compnetworking.about.com/cs/bluetooth/g/bldef_blu etooth.htm

[7]. http://www.bbc.co.uk/webwise/guides/about-bluetooth [8].http://www.engineersgarage.com/articles/bluetooth-

Technology

[9].http://en.wikipedia.org/wiki/Near_field_communication [10].http://www.nearfieldcommunication.org/technology. html

[11].http://www.pcadvisor.co.uk/how-to/mobilephone/3472879/what-is-nfc-how-nfc-works-what-it-does/

\section{BIOGRAPHIES}

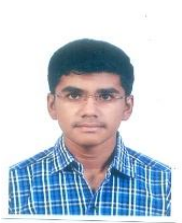

R.Rajesh currently a student at Narasu's Sarathy Institute Of Technology, I am a member of Computer Society Of India. My research interested on Hardware Security

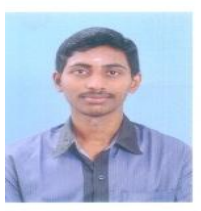

P.Dhanaraja currently a student at Narasu's Sarathy Institute Of Technology, I am a member of Computer Society Of India. My research interested on Hardware Security.

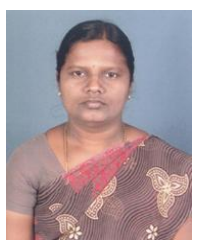

A.Gomathi, M.E (Ph.D), Associate Professor, at Narasu's Sarathy Institute Of Technology, Doing Ph.D in Anna University, Won the "Best paper award "in National Conference at Hindustan College of Engineering, Coimbatore. 